Original Article

\title{
Triptolide improves myocardial fibrosis in rats through inhibition of nuclear factor kappa B and NLR family pyrin domain containing 3 inflammasome pathway
}

\author{
Jianyao Shen, Hailiang Ma, and Chaoquan Wang* \\ Department of Cardiology, The Central Hospital Affiliated to Shaoxing University, Shaoxing 312030, China
}

\author{
ARTICLE INFO \\ Received April 19, 2021 \\ Revised July 5, 2021 \\ Accepted July 7, 2021 \\ *Correspondence \\ Chaoquan Wang \\ E-mail: chaoqw0825@163.com
}

\section{Key Words}

Endomyocardial fibrosis

Heart function tests

$\mathrm{NF}-\kappa \mathrm{B}$ pathway

NLRP3 inflammasome

Triptolide

\begin{abstract}
Myocardial fibrosis (MF) is the result of persistent and repeated aggravation of myocardial ischemia and hypoxia, leading to the gradual development of heart failure of chronic ischemic heart disease. Triptolide (TPL) is identified to be involved in the treatment for MF. This study aims to explore the mechanism of TPL in the treatment of MF. The MF rat model was established, subcutaneously injected with isoproterenol and treated by subcutaneous injection of TPL. The cardiac function of each group was evaluated, including LVEF, LVFS, LVES, and LVED. The expressions of ANP, BNP, inflammatory related factors (IL-1 $\beta$, IL-18, TNF- $\alpha$, MCP-1, VCAM1), NLRP3 inflammasome factors (NLRP3, ASC) and fibrosis related factors (TGF- $\beta 1$, COL1, and COL3) in rats were dete cted. H\&E staining and Masson staining were used to observe myocardial cell inflammation and fibrosis of rats. Western blot was used to detect the p-P65 and t-P65 levels in nucleoprotein of rat myocardial tissues. LVED and LVES of MF group were significantly upregulated, LVEF and LVFS were significantly downregulated, while TPL treatment reversed these trends; TPL treatment downregulated the tissue injury and improved the pathological damage of MF rats. TPL treatment downregulated the levels of inflammatory factors and fibrosis factors, and inhibited the activation of NLRP3 inflammasome. Activation of NLRP3 inflammasome or NF- $\kappa$ B pathway reversed the effect of TPL on MF. Collectively, TPL inhibited the activation of NLRP3 inflammasome by inhibiting NF-אB pathway, and improved MF in MF rats.
\end{abstract}

\section{INTRODUCTION}

Myocardial fibrosis (MF) is defined as various quantitative and qualitative changes of the myocardial interstitial collagen network which occurs in response of cardiac ischemic injuries, systemic diseases, drugs, or any other harmful stimulation affecting the circulatory system or the heart itself [1]. MF is a common phenomenon in the late stage of many heart diseases and is a predictor of sudden cardiac death [2], which may be caused by a series of pathological processes [3]. Myocardial fibroblasts become pro- inflammatory cells and activating the inflammasome in the early stages of infarct healing [4]. Evidence also supports the effect of inflammation on the initiation and promotion of diverse cardiovascular diseases, including MF [5]. MF changes the myocardium architecture, leads to cardiac dysfunction, arrhythmias and ischemia, and thus influences the clinical course and prognosis of patients with heart failure [6]. However, there still lacks satisfactory treatment for MF and further work is needed for the effective intervention of MF.

Triptolide (TPL) (Fig. 1) is a main active component extracted
This is an Open Access article distributed under the terms of the Creative Commons Attribution Non-Commercial License, which permits unrestricted non-commercial use, distribution, and reproduction in any medium, provided the original work is properly cited. Copyright $\odot$ Korean J Physiol Pharmacol, pISSN 1226-4512, elSSN 2093-3827
Author contributions: J.Y.S. is the guarantor of integrity of the entire study; J.Y.S. contributed to the study concepts, study design, definition of intellectual content, literature research, manuscript preparation and manuscript editing and review; H.L.M. contributed to the clinical studies; J.Y.S. and C.Q.W. contributed to the experimental studies and data acquisition; C.Q.W. contributed to the data analysis and statistical analysis. All authors read and approved the final manuscript. 


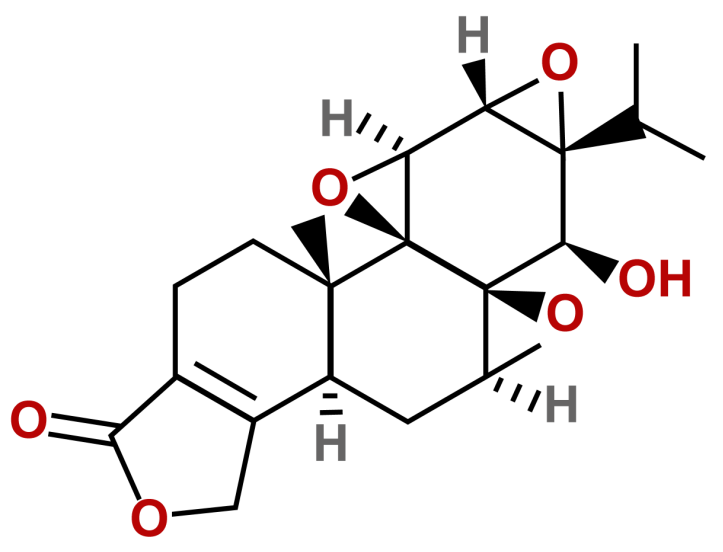

Fig. 1. Chemical structure of TPL. TPL, triptolide.

from traditional Chinese herbal medicine Tripterygium wilfordii, which exhibits multiple pharmacological effects [7], including potent antitumor, immunosuppressive, and anti-inflammatory properties, but the effects are limited in clinic because of the poor solubility, toxicity and bioavailability [8]. The value of TPL in the treatment of various inflammatory disorders and cancers has been emphasized over the past few years $[9,10]$. Evidence has also shown that the TPL plays a role in attenuating cardiac fibrosis [11]. Inflammatory is one of the best-studied mediators related to cardiac fibrosis [12]. The NLRP3 inflammasome is noticed as a key to regulate metabolic inflammation [13]. The inhibitor of NLRP3 can alleviate the MF [14]. It is reported that an appropriate dose of TPL can inhibit the activation of NLRP3 inflammasome [15]. It is shown that TPL can downregulate NLRP3 [16]. Collectively, TPL may play an effective role in interrupting the activation of NLRP3 inflammasome to improve MF.

At present, there are few reports on the effect of TPL on treating MF induced by isoproterenol (ISO), and the mechanism of TPL improving the condition of MF needs to be further explored. This study investigated the protective mechanism of TPL on MF rats, and shall provide a new theoretical basis for the improvement of TPL on MF.

\section{METHODS}

\section{Ethics statement}

All procedures were authorized by the academic ethics committee of The Central Hospital Affiliated to Shaoxing University. All procedures were strictly implemented by the guidelines of Animal Care Committee of The Central Hospital Affiliated to Shaoxing University. All the laboratory procedures were used to reduce the pain of the rats, such as heating pads, disinfection and replenishing fluids with saline.

\section{Laboratory animals and treatment}

Adult male Sprague-Dawley (SD) rats, 8-10 weeks old, weighing 220-270 g, were purchased from Shanghai Shrek experimental animal Co., Ltd. (Shanghai, China). The rats were raised in a clean room in a 12 -h light/dark cycle at $20^{\circ} \mathrm{C}$ with $50 \%$ and $60 \%$ humidity. All animals were euthanized by intraperitoneal injection of pentobarbital sodium $\geq 100 \mathrm{mg} / \mathrm{kg}$.

All the SD rats were randomly assigned into 8 groups: sham group (subcutaneously injected with $5 \mathrm{mg} / \mathrm{kg} / \mathrm{d}$ saline), MF group (subcutaneously injected with $5 \mathrm{mg} / \mathrm{kg} / \mathrm{d}$ isoproterenol, ISO), dimethyl sulfoxide (DMSO) group (subcutaneously injected with ISO at $5 \mathrm{mg} / \mathrm{kg} / \mathrm{d}$ and DMSO at $200 \mu \mathrm{g} / \mathrm{kg} / \mathrm{d}$ ) and TPL group (subcutaneously injected with ISO at $5 \mathrm{mg} / \mathrm{kg} / \mathrm{d}$ and intraperitoneal injected with TPL at $200 \mu \mathrm{g} / \mathrm{kg} / \mathrm{d}$ ) (Sigma, St. Louis, MO, USA) for 7 days $[17,18]$. The heart function was measured 7 days after injection and following experiments were carried out. In the joint experiment, rats in TPL group were intraperitoneally injected with NLRP3 inflammasome activator Nigericin $(2.5 \mathrm{mg} /$ $\mathrm{kg} / \mathrm{d})$ (Sigma) or NF- $\mathrm{B}$ pathway activator Jaceosidin $(2.5 \mathrm{mg} / \mathrm{kg} /$ d) (Sigma) for 7 days [19,20], and the injection of phosphate buffer saline (PBS) was used as the control group.

\section{Cardiac function test}

After 7 days of continuous injection, echocardiography was performed to evaluate cardiac function. The rats were anesthetized by an intraperitoneal injection of pentobarbital sodium (40 $\mathrm{mg} / \mathrm{kg}$ ) and fixed on the examination table in supine position. The chest hair was removed and the electrocardiogram was recorded simultaneously. The left ventricular end systolic diameter (LVES) and left ventricular end diastolic diameter (LVED) were measured by M-mode echocardiography images in parasternal long axis view of left ventricle. The system calculated the left ventricular ejection fraction (LVEF) and left ventricular fractional shortening (LVFS) automatically. Vevo 2100 small animal ultrasonograph (Fujifilm VisualSonics, Inc., Toronto, ON, Canada) was used for analysis.

\section{Sample collection}

All animals were euthanized by an intraperitoneal injection of pentobarbital sodium $\geq 100 \mathrm{mg} / \mathrm{kg}$ after cardiac function test [21]. The myocardial tissues of some rats were taken out and embedded in paraffin and each specimen was cut into sections at $4 \mu \mathrm{m}$ for histological experiments. The myocardial tissues of the rest rats were made into homogenate for subsequent detection.

\section{Immunohistochemistry}

The prepared myocardial tissues of rats were sectioned, dewaxed, repaired in antigen repair solution, added normal goat 
serum sealing solution (C-0005; Shanghai Haoran Biotechnology Co., Ltd., Shanghai, China), and placed at room temperature for $20 \mathrm{~min}$. Next, the sections were added primary antibodies rabbit anti-mouse NLRP3 (ab214185, 1:200; Abcam, Cambridge, MA, USA), ASC (ab47092, 1:100; Abcam), TGF- $\beta 1$ (ab215715, 1:500; Abcam), COL1 (ab3453, 1:100; Abcam) and COL3 (ab23445, 1:100; Abcam) overnight at $4^{\circ} \mathrm{C}$. After that, the sections were added goat anti-rabbit secondary $\operatorname{IgG}(1: 1,000, \mathrm{ab} 6785 ; \mathrm{Abcam})$ at $37^{\circ} \mathrm{C}$ for 20 min, stained by 2,4-diaminobutyric acid (ST033; Guangzhou Weijia Technology Co., Ltd., Guangzhou, China), counterstained by hematoxylin (PT001; Shanghai Bogu Biotechnology Co., Ltd., Shanghai, China) for $1 \mathrm{~min}$, and returned to blue by $1 \%$ ammonia. Finally, the sections were sealed by neutral resin, and observed and photographed under the microscope (Olympus, Tokyo, Japan). Five visual fields were randomly selected, and the proportion of the average positive area in the five visual fields was used to quantitatively evaluate the expression of fibrosis related factors.

\section{Hematoxylin and eosin (H\&E) staining}

The rat hearts were soaked in $4 \%$ paraformaldehyde for $24 \mathrm{~h}$, and each specimen was cut into sections at $4 \mu \mathrm{m}$. H\&E staining was performed after dewaxing. The sections were stained with hematoxylin for $5 \mathrm{~min}$ and stained with eosin for $5 \mathrm{~s}$. Then sections were then dehydrated, cleared, and sealed by neutral resin and finally the pathological damage of rats was observed by microscope.

\section{Masson staining}

The prepared myocardial tissue sections were treated with Masson staining solution (Sigma), 1\% phosphotungstic acid solution, aniline blue solution, and 1\% glacial acetic acid solu- tion successively, and images were acquired and analyzed after dehydration and clearance. The collagen fibers were stained blue, the nucleus was stained blue, and the cytoplasm was stained red. Blind method was used to analyze 5 visual fields in each section and the blue area of collagen fibers. The collagen volume fraction $(\mathrm{CVF})$ was measured. CVF $=$ the blue area of collagen fibers/full field area $\times 100 \%$, and the mean value was calculate.

\section{Reverse transcription quantitative polymerase chain reaction ( $R T-q P C R)$}

Total RNA of myocardial tissue homogenate was respectively extracted by RNAiso Plus (TAKARA, Otsu, Japan) and Trizol LS Regent (TAKARA). The reliability of extracted RNA was verified by formaldehyde denaturation electrophoresis. Subsequently, the reverse transcription experiment was performed with PrimeScript RT kit (TAKARA) in strict accordance with the instructions. SYBR Premix Ex Taq (TAKARA) was used to quantify the relative expression of genes by RT-qPCR. GAPDH was used as reference gene. The primers in each group were shown in Table 1.

\section{Western blot (WB) analysis}

The appropriate amount of tissue samples were cut up. Radioimmunoprecipitation assay lysate containing protease inhibitor (Beyotime, Shanghai, China) was used to lyse and extract protein. Protein concentration was determined by a bicinchoninic acid kit (Beyotime). The protein used for p-P65 detection was the nucleoprotein extracted from tissues using EpiQuik Nuclear Extraction kit (AmyJet Scientific Inc., Wuhan, China) according to the manufacturer's instructions. The protein was denatured at $100^{\circ} \mathrm{C}$ for $5 \mathrm{~min}$, separated by sodium dodecyl sulfate polyacrylamide gel electrophoresis, and transferred to polyvinylidene fluoride (PVDF) membranes (Millipore, Bedford, MA, USA).

Table 1. Primer sequence

\begin{tabular}{|c|c|c|}
\hline Gene & Forward & Reverse \\
\hline ANP & TTCTTCCTCGTCTTGGCСTTT & GACСТСАТСТTСТАССGGСАTСТ \\
\hline$B N P$ & CACCGCTGGGAGGTCACT & GTGAGGCСTTGGTCСTTCAA \\
\hline$I L-1 \beta$ & СССTGCAGCTGGAGAGTGTGG & ACGTGAGCAAGATCTTCCCA \\
\hline IL-18 & ACGTGAGCAAGATCTTCCCA & CСССАGAAGTCATCGTCAGTG \\
\hline$T N F-\alpha$ & TGAAGGACAAACCGAAGG & GGAAGAGCAGAAAGCGATA \\
\hline$M C P-1$ & САGАТСТСТСТTССТССАССАСТАТ & CAGGCAGCAACTGTGAACAAC \\
\hline VCAM-1 & CTCAGGTGGCTGCACAAGTT & AGAGCTCAACACAAGCGTGG \\
\hline NLRP3 & GTTTGACCCCGATGATGAGC & CTTGTGGATGGGTGGGTTTG \\
\hline ASC & TGAAGGACAAACCGAAGG & GGAAGAGCAGAAAGCGATA \\
\hline$T G F-\beta 1$ & СССТGCAGCTGGAGAGTGTGG & TGTGCTCTGCTTGAGAGGTGCT \\
\hline COL1 & GСTCСTCTTAGGGGCCACT & CCACGTCTCACCATTGGGG \\
\hline COL3 & CTGTAACATGGAAACTGGGGAAA & ССАТАGСТGAАСТGAAAACСАСC \\
\hline GAPDH & AAGAAGGTGGTGAAGCAGGC & TCCACCACCCAGTTGCTGTA \\
\hline
\end{tabular}

ANP, atrial natriuretic peptide; BNP, brain natriuretic peptide; MCP-1, monocyte chemoattractant protein 1; VCAM-1, vascular cell adhesion molecule 1; ASC, apoptosis associated speck-like protein containing a CARD domain; IL, interleukin; GAPDH, glyceraldehyde3-phosphate dehydrogenase. 
The membranes were incubated with buffer at $4^{\circ} \mathrm{C}$ for $2 \mathrm{~h}$, and added with rabbit anti-mouse primary antibodies IL-1 $\beta$ (1:1,000, ab234437; Abcam), IL-18 (1:1,000, ab207323; Abcam), TNF- $\alpha$ (1:1,000, ab215188; Abcam), NLRP3 (1:500, ab270449; Abcam), ASC (1:1,000, ab47092; Abcam), and p-P65 (1:1,000, ab194726; Abcam) at $4^{\circ} \mathrm{C}$ overnight. After three times of cleaning of PVDF membranes with tris buffered saline and Tween-20 (TBST), the PVDF membranes were incubated with anti-rabbit IgG secondary antibody $(1: 2,000, \mathrm{ab} 6721 ; \mathrm{Abcam}) 4^{\circ} \mathrm{C}$ for $4 \mathrm{~h}$. The bands were visualized using chemiluminescence and analyzed by gray analysis, and GAPDH was the control of total protein content and Lamin B1 (1 $\mu \mathrm{g} / \mathrm{ml}$, ab16048; Abcam) was the internal reference of nucleoprotein.

\section{Statistical analysis}

SPSS 21.0 statistical software (IBM Co., Armonk, NY, USA) was used to analyze data. Kolmogorov-SmiRnov test showed that the data were in normal distribution and expressed as mean \pm standard deviation. Independent sample t-test was used for comparison between two groups and one-way analysis of variance (ANOVA) was used for comparison among multi-groups. Tukey's multiple comparisons test was used for post-hoc test. p-value was obtained by a bilateral test, where $\mathrm{p}<0.05$ indicated statistical significance and $\mathrm{p}<0.01$ indicated a very significant difference.

\section{RESULTS}

\section{TPL improved cardiac dysfunction in MF rats}

TPL is the main active component of tripterygium wilfordii hook, which has been reported for many times that for the functions of anti-inflammation, anti-tumor and immunosuppression [9]. However, the role of TPL in MF rats remains to be further studied. One week after ISO induction, the cardiac function of rats was measured by small animal ultrasonograph. Compared with sham-operated rats, LVED and LVES of rats in MF group and DMSO group were significantly upregulated, while LVEF and LVFS were significantly decreased, which indicated that the systolic and diastolic functions of rat hearts induced by ISO were significantly impaired, and cardiac dysfunction existed, while TPL treatment effectively improved the cardiac dysfunction of MF rats (all p $<0.05$, Fig. 2A-D). Atrial natriuretic peptide (ANP) and brain natriuretic peptide (BNP) are secreted by ventricular myocytes, and their concentrations are closely related to cardiac
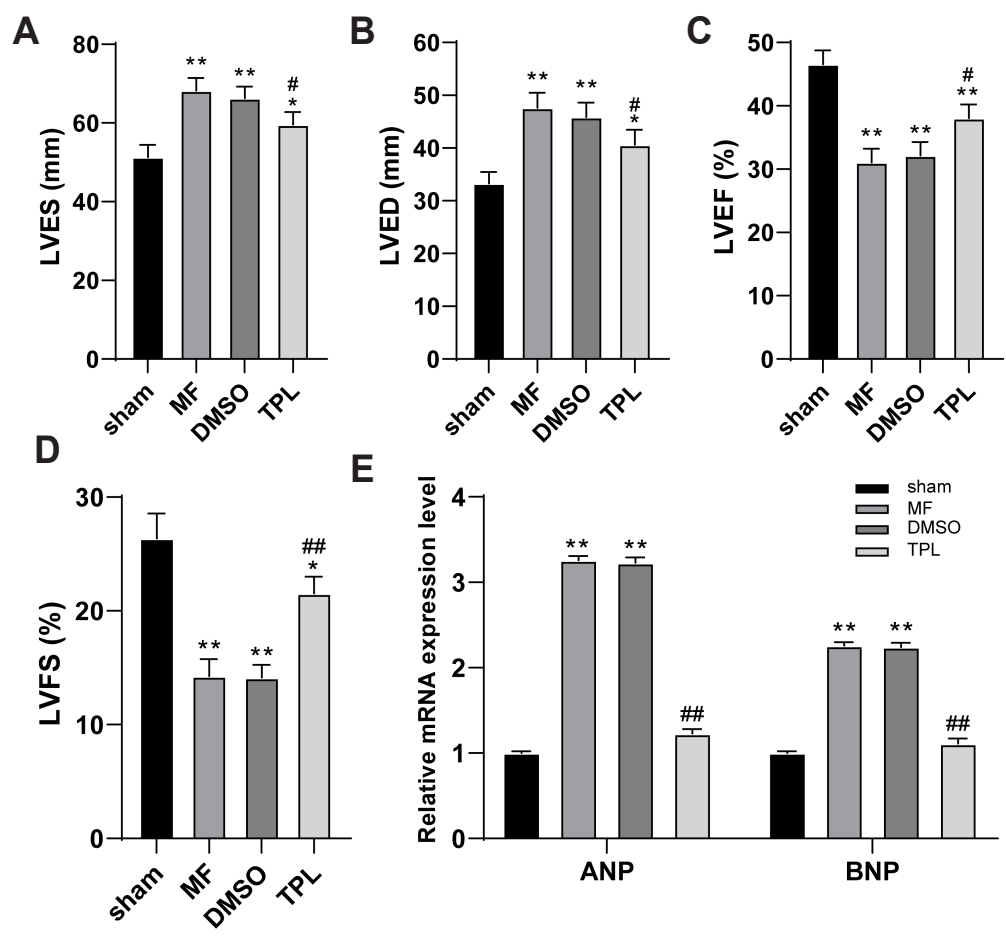

E

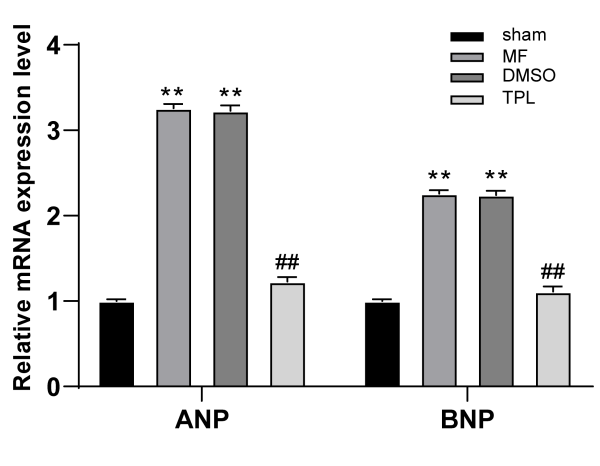

Fig. 2. TPL improved cardiac dysfunction in MF rats. Myocardial fibrosis model was established by subcutaneous injection of ISO, and TPL was injected subcutaneously for 7 days. (A-D) The LVEF, LVFS, LVES and LVED were measured by the small animal ultrasonograph; (E) RT-qPCR was used to detect the ANP and BNP levels in myocardial tissues of rats in each group; $n=6$. Three independent repeated tests were performed and the data were expressed as mean \pm SD; one-way was used for variance analysis; Tukey's multiple comparisons test was used for post-hoc test. TPL, triptolide; MF, myocardial fibrosis; DMSO, dimethyl sulfoxide; ISO, isoproterenol; LVEF, left ventricular ejection fraction; LVFS, left ventricular fractional shortening; LVES, left ventricular end systolic diameter; LVED, left ventricular end diastolic diameter; ANP, atrial natriuretic peptide; BNP, brain natriuretic peptide. Compared with sham group, ${ }^{*} \mathrm{p}<0.05,{ }^{* *} \mathrm{p}<0.01$; compared with MF group, ${ }^{\#} \mathrm{p}<0.05,{ }^{\# *} \mathrm{p}<0.01$. 
function $[9,22]$. Therefore, the mRNA expressions of ANP and $\mathrm{BNP}$ in rat myocardium were measured. The results showed that the levels of ANP and BNP in MF group and DMSO group were higher than those in sham group, while the levels of ANP and BNP in TPL group were significantly decreased (all $p<0.01$, Fig. $2 \mathrm{E})$. These results suggested that TPL could effectively improve cardiac dysfunction in MF rats.

\section{TPL reduced the degree of MF and fibrosis in MF rats}

MF could cause myocardial tissue disorder and large amount of collagen deposition [23]. Therefore, H\&E staining and Masson staining were used to observe the pathological morphology and MF of rats in each group. H\&E staining showed that compared with sham group, the disorder of myocardial tissue structure and injury of MF group and DMSO group were significantly aggravated, while the tissue injury score of TPL group was effectively decreased $(\mathrm{p}<0.01$ ), which indicated that TPL treatment could improve degree of the pathological damage of MF rats. Masson staining demonstrated that there were abundant collagen fibers in the rats of MF group and DMSO group (Fig. 3A). CVF quantita- tive analysis indicated that the collagen volume fraction of rats in MF group and DMSO group was significantly increased relative to that of sham group, and TPL treatment significantly reduced CVF of rats ( $p<0.01$, Fig. 3B). RT-qPCR was used to detect the expression of fibrosis related factors. Expressions of fibrosis related factors TGF- $\beta 1$, COL1, and COL3 in MF group and DMSO group were higher than that in sham group, while those in TPL group were lower than that in MF group (all $\mathrm{p}<0.01$, Fig. 3 C). The results of immunohistochemistry were consistent (all $\mathrm{p}<0.01$, Fig. 3D). These results suggested that TPL could downregulate the degree of MF and the expression of fibrosis related factors in MF rats.

\section{TPL downregulated the level of inflammatory factors in MF rats}

Cardiac dysfunction was often accompanied by inflammation $[24,25]$. The mRNA and protein levels of inflammatory factors IL$1 \beta$, IL-18, and TNF- $\alpha$ in myocardial tissue of rats were detected in each group. Compared with sham group, the mRNA and protein levels of inflammatory factors IL-1 $\beta$, IL-18, and TNF- $\alpha$ in MF
A

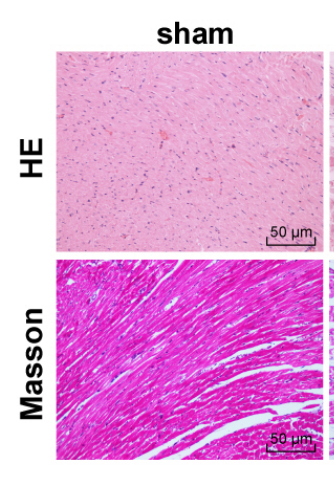

MF

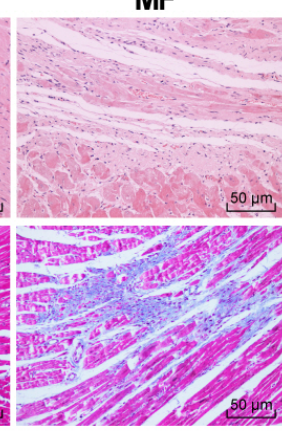

DMSO

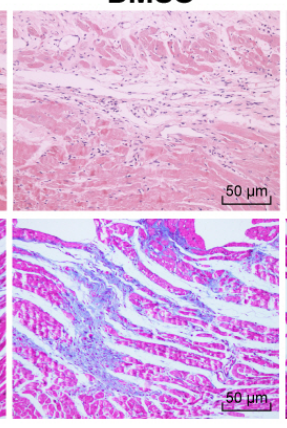

TPL

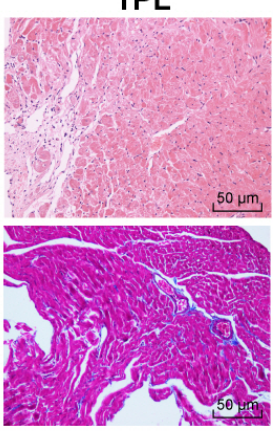

B

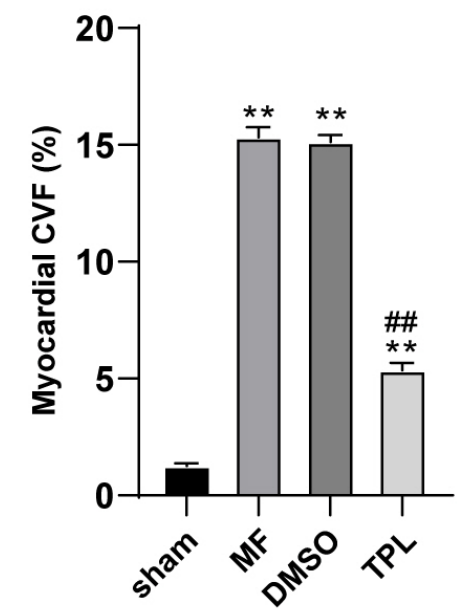

C

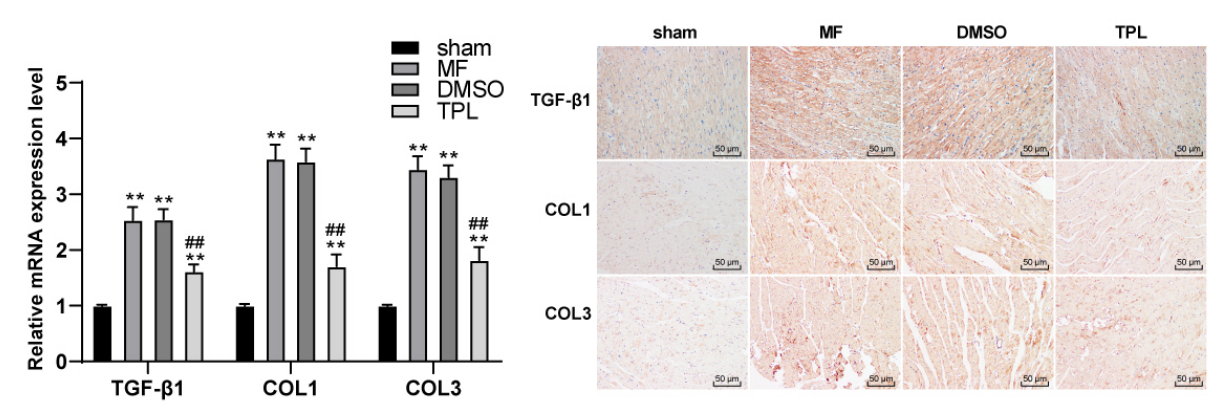

D

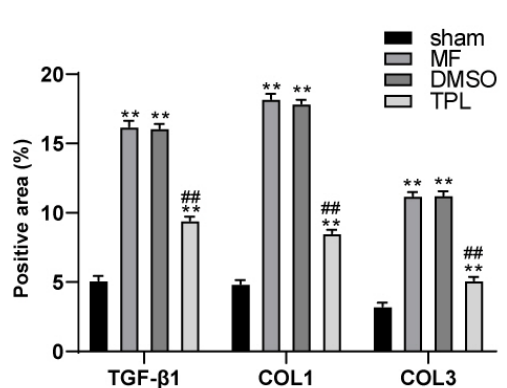

Fig. 3. TPL downregulated the degree of MF and the expression of fibrosis related factors in MF rats. (A) H\&E staining and Masson staining were used to observe the pathological morphology and MF of rats in each group ( $\times 200)$; (B) Masson staining results were quantitatively analyzed by measuring collagen volume fraction; (C) RT-qPCR was used to detect the expressions of TGF- $\beta 1$, COL1, and COL3; (D) The expressions of TGF- $\beta 1$, COL1, and $\mathrm{COL3}$ were detected by immunohistochemistry; $\mathrm{n}=6$. Three independent repeated tests were performed and the data were expressed as mean $\pm \mathrm{SD}$; one-way was used for variance analysis; Tukey's multiple comparisons test was used for post-hoc test. TPL, triptolide; MF, myocardial fibrosis; DMSO, dimethyl sulfoxide. Compared with sham group, ${ }^{* *} \mathrm{p}<0.01$; compared with MF group, ${ }^{\#} \mathrm{p}<0.05,{ }^{\# \#} \mathrm{p}<0.01$. 
A

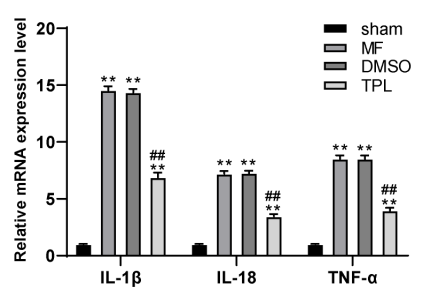

B

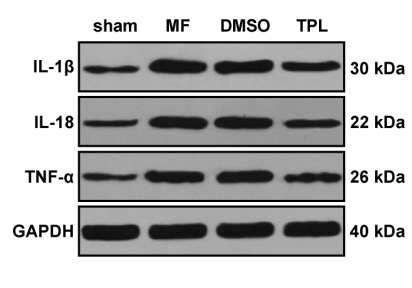

C

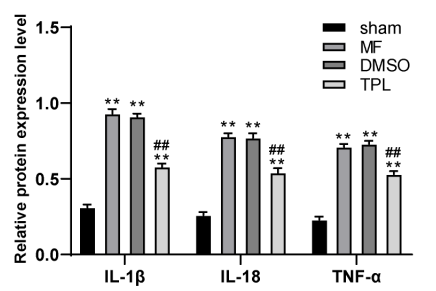

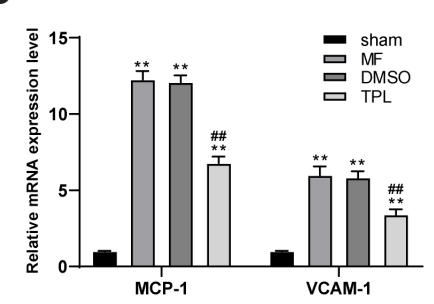

Fig. 4. TPL downregulated the level of inflammatory factors in MF rats. (A, B) RT-qPCR and WB were used to respectively detect the mRNA and protein levels of inflammatory factors IL-1 $\beta$, IL-18 and TNF- $\alpha$ in the myocardium of rats; (C) RT-qPCR was used to detect the mRNA expression of MCP1 and VCAM-1 in myocardium of rats in each group; $\mathrm{n}=6$. Three independent repeated tests were performed and the data were expressed as mean \pm SD; one-way ANOVA was used for variance analysis; Tukey's multiple comparisons test was used for post-hoc test. TPL, triptolide; MF, myocardial fibrosis; WB, Western blot; MCP-1, monocyte chemoattractant protein 1; VCAM-1, vascular cell adhesion molecule 1; DMSO, dimethyl sulfoxide. Compared with sham group, ${ }^{* *} \mathrm{p}<0.01$; compared with MF group, ${ }^{\# \#} \mathrm{p}<0.01$.

A

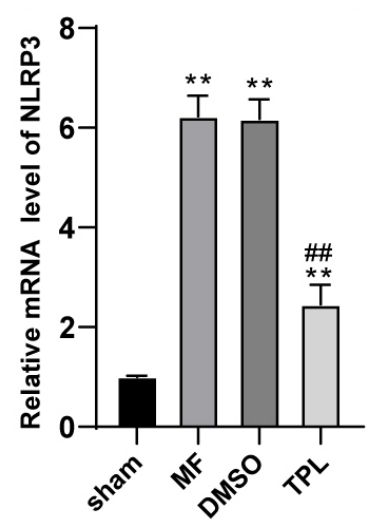

D

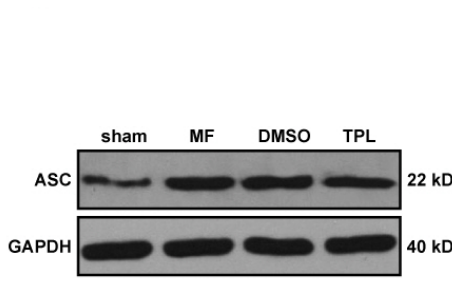

B

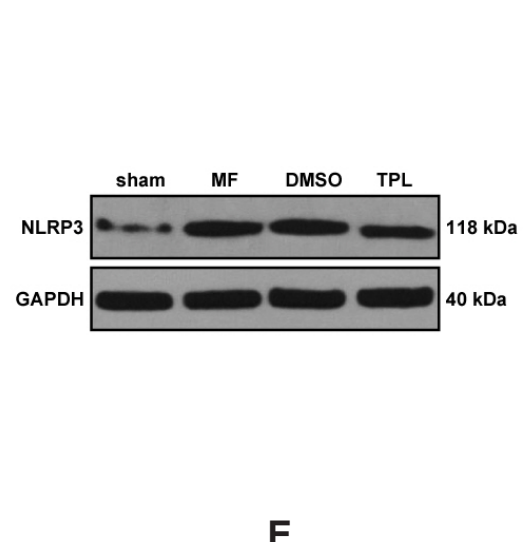

E
C

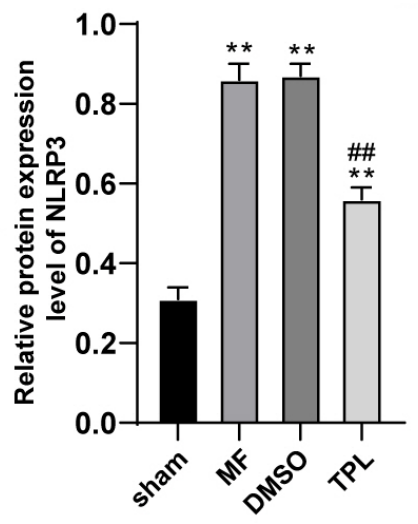

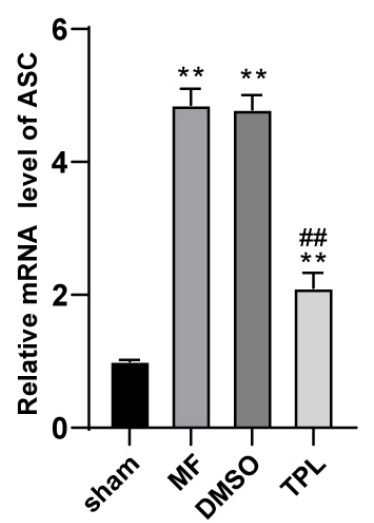
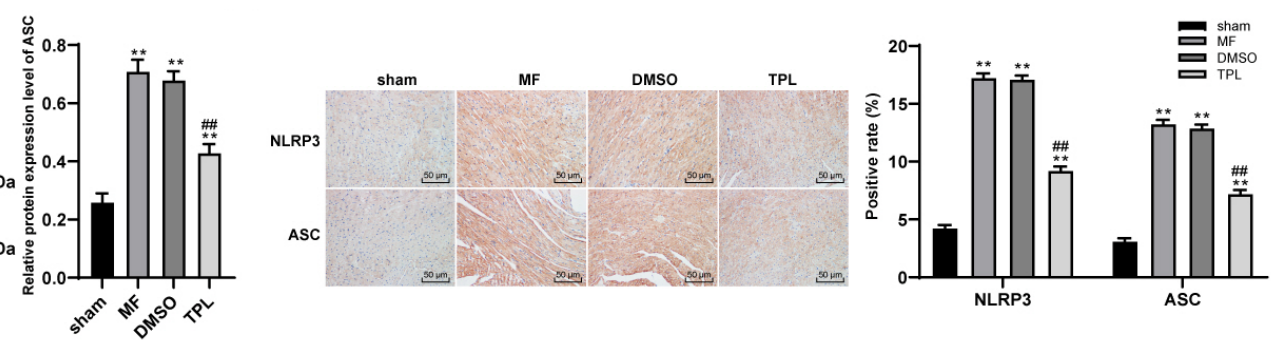

Fig. 5. TPL inhibited the activation of NLRP3 inflammasome in MF rats. (A-D) RT-PCR and WB were used to detect the mRNA and protein levels of NLRP3 and ASC in MF rats; (E) The expressions of NLRP3 and ASC were detected by immunohistochemistry $(\times 200) ; n=6$. Three independent repeated tests were performed and the data were expressed as mean \pm SD; one-way ANOVA was used for variance analysis; Tukey's multiple comparisons test was used for post-hoc test. TPL, triptolide; MF, myocardial fibrosis; WB, Western blot; DMSO, dimethyl sulfoxide. Compared with sham group, ${ }^{* *} \mathrm{p}<0.01$; compared with MF group, ${ }^{\# \#} \mathrm{p}<0.01$.

group and DMSO group were significantly upregulated, while TPL treatment effectively decreased the expression of inflammatory factors (all $\mathrm{p}<0.01$, Fig. 4A, B). Monocyte chemoattractant protein 1 (MCP-1) and vascular cell adhesion molecule 1 (VCAM1) are also key inflammatory factors, which could promote the occurrence of inflammatory reaction [26,27]. The mRNA expressions of MCP-1 and VCAM-1 were also detected, and the results were consistent with our predicted trend. TPL treatment could downregulate the expression of MCP-1 and VCAM-1 in MF rats (all $\mathrm{p}<0.01$, Fig. 4C). These results suggested that TPL could downregulate the inflammatory level of MF rats.

\section{TPL inhibited the activation of NLRP3 inflammasome in MF rats}

Apoptosis associated speck-like protein containing a CARD domain (ASC) is oligomerized by six $\alpha$-helix structure, which could recruit and activate other proteins with homologous domains and played an important biological role in inflammatory response [28]. ASC is essentially involved in the formation of NLRP3 inflam- 
masome. RT-qPCR and WB were used to respectively detect the mRNA and protein levels of NLRP3 and ASC. Compared with sham group, the mRNA and protein levels of NLRP3 and ASC in MF group and DMSO group were significantly upregulated, and TPL treatment effectively downregulated their expressions (all $\mathrm{p}<0.01$, Fig. 5A-D). The results of immunohistochemistry were consistent (Fig. 5E). It was suggested that TPL inhibited the activation of NLRP3 inflammasome in MF rats.

\section{TPL improved MF in rats by inhibiting the activation of NLRP3 inflammasome pathway}

It was confirmed through previous experiments that TPL could inhibit the inflammasome of NLRP3 and improve the MF. Therefore, we speculated that TPL might improve the MF by regulating the NLRP3 inflammatory pathway. In order to verify the conjecture, a joint experiment was conducted: NLRP3 inflammasome activator Nigericin was added in TPL-treated group, with the addition of PBS as control group. The changes of MF degree in MF rats were observed. The mRNA expressions of inflammatory factors IL-1 $\beta$, IL-18 and TNF- $\alpha$ in TPL + Nigericin group were higher than those in control group (all $p<0.01$, Fig. 6A), indicating that NLRP3 pathway was successfully interfered, and the inflammatory level of TPL + Nigericin group was upregulated. Subsequently, the mRNA expressions of fibrosis related factors (TGF- $\beta 1$, COL1, and COL3) were detected by RT-qPCR. The mRNA expressions of fibrosis related factors in TPL + Nigericin group were significantly higher than that in TPL + PBS group (all $p<0.01$, Fig. 6B). The results of immunohistochemistry were consistent (Fig. 6C). These results suggested that TPL improved the degree of MF in MF rats by inhibiting the activation of NLRP3 inflammasome pathway.
TPL inhibited the activation of NLRP3 inflammasome by inhibiting NF-kB pathway and thus improving the MF in rats

NLRP3 pathway is an important downstream pathway of NF$\kappa \mathrm{B}$ pathway, and NF- $\kappa \mathrm{B}$ pathway is often reported to be associated with inflammation and MF $[29,30]$. It was speculated that TPL might inhibit the activation of NLRP3 inflammasome by inhibiting the NF- $\mathrm{B}$ signaling pathway and improve the degree of MF. Therefore, the expression changes of the NF- $\kappa \mathrm{B}$ pathway-related proteins p-P65 and t-P65 in the nucleoprotein of myocardial tissues were detected, and it was found that P65 phosphorylation levels in MF group and DMSO group were higher than that in sham group, while TPL treatment effectively downregulated P65 phosphorylation level ( $p<0.01$, Fig. 7A). Subsequently, the NF$\kappa \mathrm{B}$ pathway activator Jaceosidin was used to treat rats in the TPL group, and the injection of PBS was used as the control group, and the changes of inflammatory and fibrosis related factors in myocardial tissue were detected by RT-qPCR. Levels of inflammatory factors and fibrosis factors in TPL + Jaceosidin group were higher than those in TPL + PBS group $(\mathrm{p}<0.01$, Fig. $7 \mathrm{~B}, \mathrm{C})$. All the above results confirmed that TPL could improve the MF by inhibiting NF- $\mathrm{KB}$ pathway and the activation of NLRP3 inflammasome.

\section{DISCUSSION}

MF, mainly caused by the death of ischemic cells in the process of myocardial infarction that leads to a reparative response in which the damaged tissue is replaced with a fibrotic scar, can lead to impairment of cardiac function and finally causes adverse outcomes of heart failure [31]. However, there is no therapeutic strategy that can ensure its reversal at present [32]. Evidences have shown that TPL has the characteristics of anti-inflammatory and

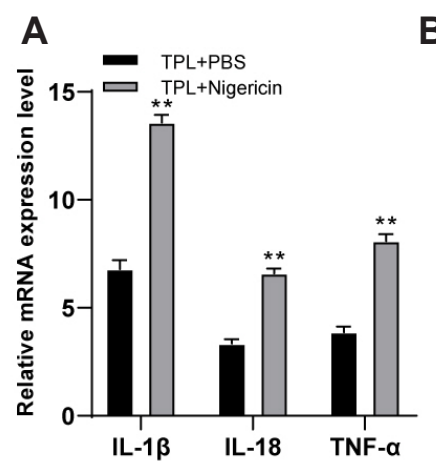

B

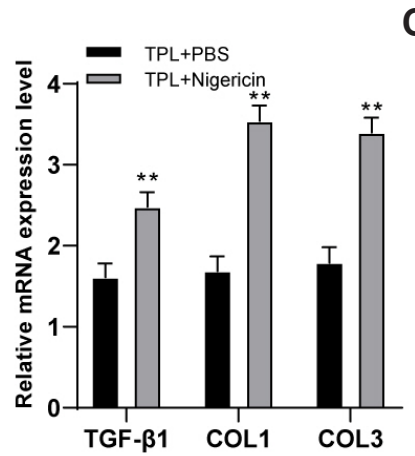

C
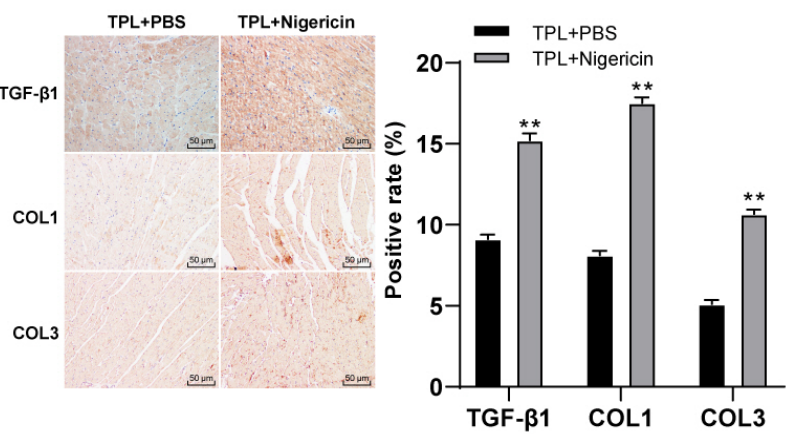

Fig. 6. TPL improved the degree of MF in MF rats by inhibiting the activation of NLRP3 inflammasome pathway. (A) RT-qPCR was used to detect the mRNA expressions of inflammatory factors IL-1 $\beta$, IL-18, and TNF- $\alpha$ in myocardial tissues; (B) RT-qPCR was used to detect the mRNA expressions of TGF- $\beta 1$, COL1, and COL3; (C) The expressions of TGF- $\beta 1$, COL1, and COL3 were detected by immunohistochemistry $(\times 200) ; n=6$. Three independent repeated tests were performed and the data were expressed as mean $\pm S D$; independent t-test was used for comparisons between $2 \mathrm{groups}$. TPL, triptolide; MF, myocardial fibrosis; PBS, phosphate buffer saline. Compared with TPL + PBS group, ${ }^{* *} \mathrm{p}<0.01$. 

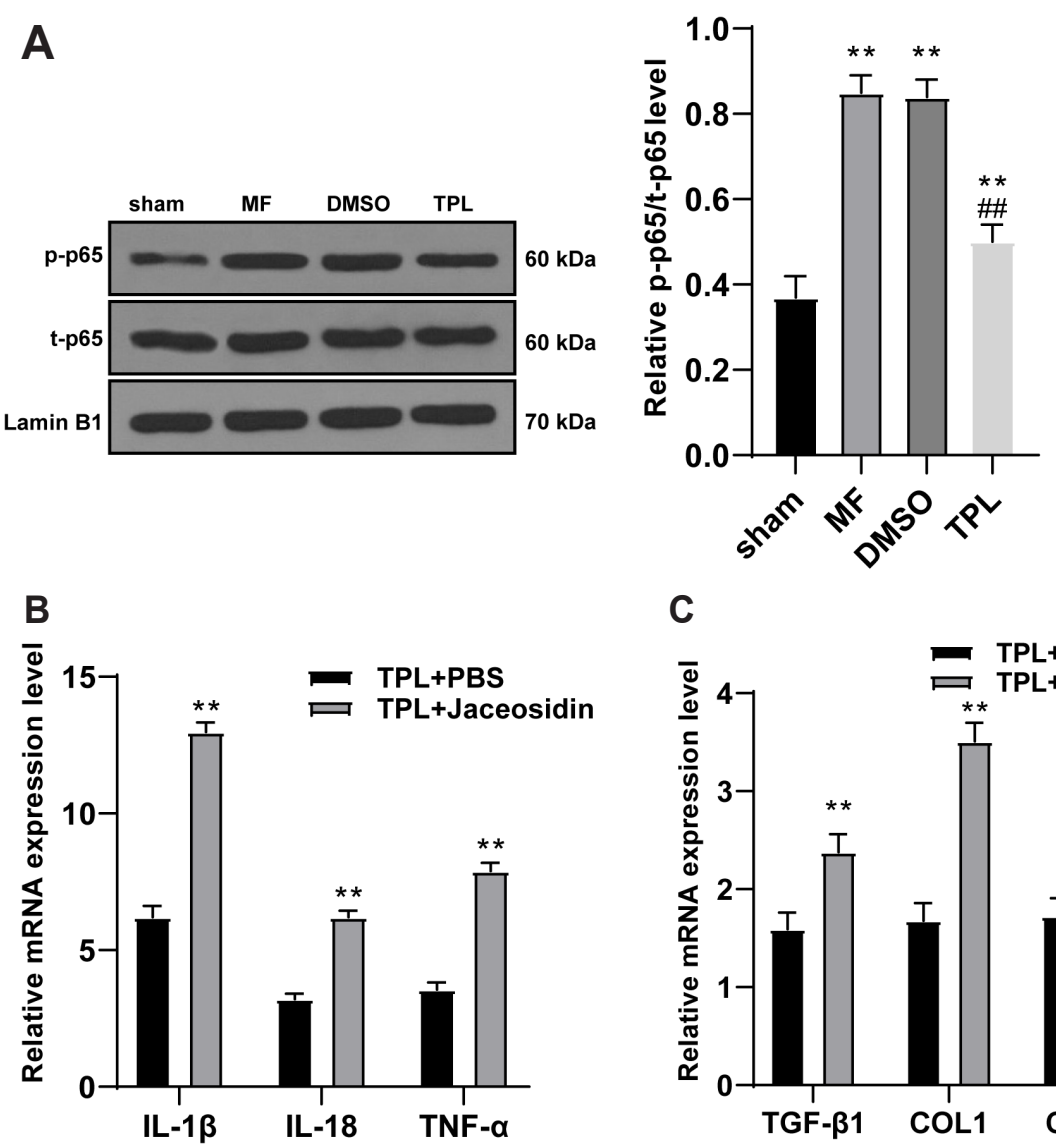

C

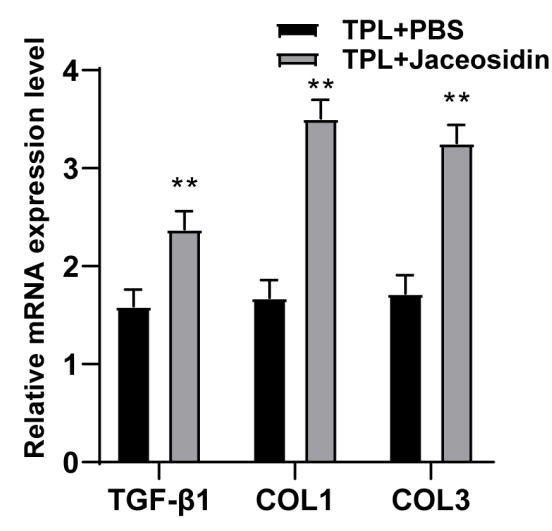

Fig. 7. TPL inhibited the activation of NLRP3 inflammasome by inhibiting NF-kB pathway and improved the degree of MF. (A) WB was used to detect the expressions of $\mathrm{p}-\mathrm{P} 65$ and t-P65 in myocardium nucleoprotein of rats in each group; (B) RT-qPCR was used to detect the mRNA expressions of inflammatory factors IL-1 $\beta$, IL-18, and TNF- $\alpha$ in myocardial tissues of rats in each group; (C) RT-qPCR was used to detect the mRNA expressions of fibrosis related factors TGF- $\beta 1, \mathrm{COL} 1$, and $\mathrm{COL} 3$ in myocardium; $\mathrm{n}=6$. Three independent repeated tests were performed and the data were expressed as mean $\pm S D$; one-way was used for variance analysis; Tukey's multiple comparisons test was used for post-hoc test. TPL, triptolide; MF, myocardial fibrosis; WB, Western blot; DMSO, dimethyl sulfoxide; PBS, phosphate buffer saline. Compared with TPL + PBS group, ${ }^{* *} \mathrm{p}<0.01$. Compared with the MF group, ${ }^{\#} p<0.01$. antitumor [9]. This study found that TPL could inhibit the activation of NLRP3 inflammasome and improve MF by inhibiting $\mathrm{NF}-\kappa \mathrm{B}$ pathway.

TPL has been shown to have potent antitumor activity in various preclinical cancer models [8], such as lung cancer, breast cancer and pancreatic cancer [33-35]. However, the effect of TPL on MF was still unclear. TPL has limited effects due to its poor solubility, toxicity and water solubility, while a newly developed water-soluble minnelide, has been recently reported to show promising activity in malignancies [36-40]. However, the clinical effect on myocardial fibrosis has not been reported at present, and the relevant studies are all carried out with TPL [11,17,41-43]. Considering that the focus of this study was to explore the mechanism of whether TPL could improve the degree of MF in MF rats by inhibiting the NF- $\kappa \mathrm{B}$ pathway and the activation of NLRP3 inflammasome. Therefore, we chose to carry out further mechanism research on the basis of existing studies, instead of using minnelide, which had unclear effects on myocardial fibrosis. MF can contribute to cardiac dysfunction in many pathophysiologic conditions [12]. The levels of LVED, LVES, LVEF and LVFS are main indexes of cardiac function [44]. This study showed that TPL improved cardiac dysfunction in MF rats. LVED and LVES were significantly upregulated, while LVEF and LVFS were significantly decreased in MF rats, indicating that the systolic and diastolic functions of rat hearts were significantly impaired; and the cardiac dysfunction of MF rats was effectively improved by TPL treatment. The concentrations of ANP and BNP are closely associated with cardiac function [22]. The levels of ANP and BNP in MF rats were significantly higher, while the levels of ANP and BNP in TPL group were significantly decreased. Consistently, it has been revealed that TPL therapy improves cardiac function through upregulation of MAPK signaling transduction [45]. In brief, TPL could effectively improve cardiac dysfunction in MF rats.

MF can cause myocardial tissue disorder and collagen deposition [23]. The collagen fibers in the rats of MF and DMSO groups were abundant. The collagen volume fraction of rats in MF and DMSO groups was increased significantly, and TPL group reduced CVF of rats significantly. The tissue injury in MF rats was upregulated significantly while that in TPL group was decreased effectively, indicating that TPL could improve pathological damage of MF rats. Triptolide inhibits collagen gel and suppresses the production of fibronectin [46]. A study has shown can alleviation effect of TPL on the diabetic renal fibrosis by inhibiting miR-1413p/PTEN/Akt/mTOR pathway [47]. This study explored the role of TPL in alleviating the fibrosis and the expression of fibrosisrelated factors. The fibrosis related factors (TGF- $\beta 1$, COL1, and COL3) expressions in MF and DMSO groups were much higher, while those in TPL group were much lower. Consistently, TPL inhibits airway smooth muscle cells malignant behaviors induced 
by TGF- $\beta 1$ significantly (PMID: 25267491). These suggested that TPL could alleviate MF and downregulate the fibrosis related factors expression in MF rats.

Cardiac dysfunction is often accompanied by inflammation [25]. Our results showed that the levels of inflammatory factors (IL-1 $\beta$, IL-18, and TNF- $\alpha$ ) in MF and DMSO groups were upregulated significantly, while TPL treatment decreased the expression of inflammatory factors effectively. It is reported that TPL can downregulate the expressions of IL-1 $\beta$ and TNF- $\alpha$ [48]. TPL attenuates inflammatory responses in endothelial cells [49]. In brief, these results suggested that TPL could downregulate the level of inflammatory in MF rats. NLRP3 inflammasome can finally produce a mature form of highly inflammatory cytokines [50]. The mRNA and protein levels of NLRP3 and ASC were increased significantly in MF and DMSO groups, while TPL treatment decreased their levels effectively. It is reported that TPL can inhibit the activation of NLRP3 inflammasome and protect against the inflammatory injury in diabetic kidney disease [15]. We speculate that TPL may improve MF by regulating NLRP3 inflammasome pathway. To test our hypothesis, we added NLRP3 inflammasome activator Nigericin in TPL group. Our results demonstrated that mRNA expressions of inflammatory factors and fibrosis related factors in TPL + Nigericin group were higher. There is a previous study supporting that TPL protects against pressure overload of hearts by inhibiting the activation of NLRP3 inflammasome [51]. Treatment with TPL exerts the antiinflammatory effects by downregulating NLRP3 inflammasome in nephritis [52]. In brief, it was suggested that TPL improved MF by inhibiting the activation of NLRP3 inflammasome pathway.

$\mathrm{NF}-\kappa \mathrm{B}$ pathway is reported to play an essential role in myocardial inflammation as a transcription factor [30]. TPL improves podocyte apoptosis and proteinuria by inhibiting NF- $\mathrm{kB} / \mathrm{GAD}$ D45B pathway [53]. The blocking of the nuclear translocation of p-P65 can protect against the adverse cardiac remodeling following myocardial infarction [54]. We deduced that TPL might inhibit NLRP3 inflammasome via inhibiting NF-кB pathway. The results showed that $\mathrm{P} 65$ phosphorylation levels in MF and DMSO groups were elevated, while TPL treatment downregulated the levels effectively. Next, we treated the TPL group with NF- $\kappa \mathrm{B}$ pathway activator Jaceosidin. The levels of inflammatory factor and fibrosis factors in TPL + Jaceosidin group were significantly enhanced. TPL may suppress inflammatory response of endothelial cells by inhibiting activation of NF- $\mathrm{BB}$ [49]. There is little research on the effect of TPL on inhibiting MF via NF- $\mathrm{B}$ pathway. Our study confirmed that TPL could improve MF by inhibiting NF- $\mathrm{KB}$ pathway and the activation of NLRP3 inflammasome.

In summary, this study supported that TPL could inhibit the activation of NLRP3 inflammasome by inhibiting NF- $\mathrm{\kappa B}$ pathway, thus improving MF. However, there are still some limitations in this study. Firstly, referring to the previous study [17], we only used a single dose of TPL for treatment, and no concentration gradient was set. It would be of great reference value to study the mechanism of TPL and its clinical application to find the best inhibitory concentration of TPL by setting TPL with different gradient dose. Furthermore, previous study has shown that TPL exposure can lead to various organ damage in animals and even humans, including liver, kidney, testis, ovary and heart, and the toxic effects of TPL on different cell types in vitro and target organs in vivo are different with different routes, doses and times of administration [55]. Due to the narrow therapeutic window of TPL, the therapeutic effect and toxicity range of TPL are very close, sometimes even overlapping. Therefore, it is necessary to further study the relationship between the efficacy and toxicology of TPL. In this study, although the TPL treatment conditions we used could effectively improve the cardiac dysfunction of MF rats, reduce the degree of MF and the expression of fibrosis-related factors in MF rats, we did not study its toxicity to liver, kidney and other organs. In the future, we will conduct further research on this to clarify the relationship between the efficacy and toxicology of TPL. In addition, whether there is a deeper regulatory mechanism between TPL and NF- $\mathrm{B}$ pathway in MF remains to be further studied. It is the future research trend to transfer the mechanism of TPL on improving MF to clinical application.

\section{CONFLICTS OF INTEREST}

The authors declare no conflicts of interest.

\section{REFERENCES}

1. Gyöngyösi M, Winkler J, Ramos I, Do QT, Firat H, McDonald K, González A, Thum T, Díez J, Jaisser F, Pizard A, Zannad F. Myocardial fibrosis: biomedical research from bench to bedside. Eur J Heart Fail. 2017;19:177-191.

2. van de Schoor FR, Aengevaeren VL, Hopman MT, Oxborough DL, George KP, Thompson PD, Eijsvogels TM. Myocardial fibrosis in athletes. Mayo Clin Proc. 2016;91:1617-1631.

3. Everett RJ, Stirrat CG, Semple SI, Newby DE, Dweck MR, Mirsadraee S. Assessment of myocardial fibrosis with T1 mapping MRI. Clin Radiol. 2016;71:768-778.

4. Shinde AV, Frangogiannis NG. Fibroblasts in myocardial infarction: a role in inflammation and repair. J Mol Cell Cardiol. 2014;70:74-82.

5. Marques MD, Nauffal V, Ambale-Venkatesh B, Vasconcellos HD, Wu C, Bahrami H, Tracy RP, Cushman M, Bluemke DA, Lima JAC. Association between inflammatory markers and myocardial fibrosis. Hypertension. 2018;72:902-908.

6. López B, González A, Ravassa S, Beaumont J, Moreno MU, San José G, Querejeta R, Díez J. Circulating biomarkers of myocardial fibrosis: the need for a reappraisal. J Am Coll Cardiol. 2015;65:24492456.

7. Wei YM, Wang YH, Xue HQ, Luan ZH, Liu BW, Ren JH. Triptolide, a potential autophagy modulator. Chin J Integr Med. 2019;25:233240.

8. Noel P, Von Hoff DD, Saluja AK, Velagapudi M, Borazanci E, Han H. 
Triptolide and its derivatives as cancer therapies. Trends Pharmacol Sci. 2019;40:327-341.

9. Viegas JSR, Praça FG, Kravicz M, Bentley MVLB. Therapeutic applications and delivery systems for triptolide. Drug Deliv Transl Res. 2020;10:1584-1600.

10. Yuan K, Li X, Lu Q, Zhu Q, Jiang H, Wang T, Huang G, Xu A. Application and mechanisms of triptolide in the treatment of inflammatory diseases-a review. Front Pharmacol. 2019;10:1469.

11. Pan XC, Liu Y, Cen YY, Xiong YL, Li JM, Ding YY, Tong YF, Liu T, Chen XH, Zhang HG. Dual role of triptolide in interrupting the NLRP3 inflammasome pathway to attenuate cardiac fibrosis. Int $J$ Mol Sci. 2019;20:360.

12. Kong P, Christia P, Frangogiannis NG. The pathogenesis of cardiac fibrosis. Cell Mol Life Sci. 2014;71:549-574.

13. Ralston JC, Lyons CL, Kennedy EB, Kirwan AM, Roche HM. Fatty acids and NLRP3 inflammasome-mediated inflammation in metabolic tissues. Annu Rev Nutr. 2017;37:77-102.

14. Gao R, Shi H, Chang S, Gao Y, Li X, Lv C, Yang H, Xiang H, Yang J, Xu L, Tang Y. The selective NLRP3-inflammasome inhibitor MCC950 reduces myocardial fibrosis and improves cardiac remodeling in a mouse model of myocardial infarction. Int Immunopharmacol. 2019;74:105575.

15. Wu W, Liu BH, Wan YG, Sun W, Liu YL, Wang WW, Fang QJ, Tu Y, Yee HY, Yuan CC, Wan ZY. [Triptolide inhibits NLRP3 inflammasome activation and ameliorates podocyte epithelial-mesenchymal transition induced by high glucose]. Zhongguo Zhong Yao Za Zhi. 2019;44:5457-5464. Chinese.

16. Qian K, Zhang L, Shi K. Triptolide prevents osteoarthritis via inhibiting hsa-miR-20b. Inflammopharmacology. 2019;27:109-119.

17. Guo X, Xue M, Li CJ, Yang W, Wang SS, Ma ZJ, Zhang XN, Wang XY, Zhao R, Chang BC, Chen LM. Protective effects of triptolide on TLR4 mediated autoimmune and inflammatory response induced myocardial fibrosis in diabetic cardiomyopathy. J Ethnopharmacol. 2016;193:333-344.

18. Wan Y, Xu L, Wang Y, Tuerdi N, Ye M, Qi R. Preventive effects of astragaloside IV and its active sapogenin cycloastragenol on cardiac fibrosis of mice by inhibiting the NLRP3 inflammasome. Eur $J$ Pharmacol. 2018;833:545-554.

19. Han C, Yang Y, Guan Q, Zhang X, Shen H, Sheng Y, Wang J, Zhou X, Li W, Guo L, Jiao Q. New mechanism of nerve injury in Alzheimer's disease: $\beta$-amyloid-induced neuronal pyroptosis. J Cell Mol Med. 2020;24:8078-8090.

20. Huang XL, Wei XC, Guo LQ, Zhao L, Chen XH, Cui YD, Yuan J, Chen DF, Zhang J. The therapeutic effects of Jaceosidin on lipopolysaccharide-induced acute lung injury in mice. J Pharmacol Sci. 2019;140:228-235.

21. Zatroch KK, Knight CG, Reimer JN, Pang DS. Refinement of intraperitoneal injection of sodium pentobarbital for euthanasia in laboratory rats (Rattus norvegicus). BMC Vet Res. 2017;13:60.

22. Špiranec Spes K, Chen W, Krebes L, Völker K, Abeßer M, Eder Negrin P, Cellini A, Nickel A, Nikolaev VO, Hofmann F, Schuh K, Schweda F, Kuhn M. Heart-microcirculation connection: effects of ANP (atrial natriuretic peptide) on pericytes participate in the acute and chronic regulation of arterial blood pressure. Hypertension. 2020;76:1637-1648

23. Li X, Zhao D, Guo Z, Li T, Qili M, Xu B, Qian M, Liang H, E X, Chege Gitau S, Wang L, Huangfu L, Wu Q, Xu C, Shan H. Over- expression of SerpinE2/protease nexin-1 contribute to pathological cardiac fibrosis via increasing collagen deposition. Sci Rep. 2016;6:37635.

24. Unudurthi SD, Nassal DM, Patel NJ, Thomas E, Yu J, Pierson CG, Bansal SS, Mohler PJ, Hund TJ. Fibroblast growth factor-inducible 14 mediates macrophage infiltration in heart to promote pressure overload-induced cardiac dysfunction. Life Sci. 2020;247:117440.

25. Zhou Z, Miao Z, Luo A, Zhu D, Lu Y, Li P, Feng X, Tan W, Wang F. Identifying a marked inflammation mediated cardiac dysfunction during the development of arthritis in collagen-induced arthritis mice. Clin Exp Rheumatol. 2020;38:203-211.

26. Kong DH, Kim YK, Kim MR, Jang JH, Lee S. Emerging roles of vascular cell adhesion molecule-1 (VCAM-1) in immunological disorders and cancer. Int J Mol Sci. 2018;19:1057.

27. Yoshimura T. The production of monocyte chemoattractant protein-1 (MCP-1)/CCL2 in tumor microenvironments. Cytokine. 2017;98:71-78.

28. He XF, Zeng YX, Li G, Feng YK, Wu C, Liang FY, Zhang Y, Lan Y, $\mathrm{Xu}$ GQ, Pei Z. Extracellular ASC exacerbated the recurrent ischemic stroke in an NLRP3-dependent manner. J Cereb Blood Flow Metab. 2020;40:1048-1060.

29. Qu S, Wang W, Li D, Li S, Zhang L, Fu Y, Zhang N. Mangiferin inhibits mastitis induced by LPS via suppressing NF-kB and NLRP3 signaling pathways. Int Immunopharmacol. 2017;43:85-90.

30. Viswanadha VP, Dhivya V, Beeraka NM, Huang CY, Gavryushova LV, Minyaeva NN, Chubarev VN, Mikhaleva LM, Tarasov VV, Aliev G. The protective effect of piperine against isoproterenolinduced inflammation in experimental models of myocardial toxicity. Eur J Pharmacol. 2020;885:173524.

31. Talman V, Ruskoaho H. Cardiac fibrosis in myocardial infarctionfrom repair and remodeling to regeneration. Cell Tissue Res. 2016;365:563-581.

32. Ravassa S, González A, Bayés-Genís A, Lupón J, Díez J. Myocardial interstitial fibrosis in the era of precision medicine. Biomarkerbased phenotyping for a personalized treatment. Rev Esp Cardiol (Engl Ed). 2020;73:248-254.

33. Jiang W, Chen M, Xiao C, Yang W, Qin Q, Tan Q, Liang Z, Liao X, Mao A, Wei C. Triptolide suppresses growth of breast cancer by targeting HMGB1 in vitro and in vivo. Biol Pharm Bull. 2019;42:892899.

34. Kim ST, Kim SY, Lee J, Kim K, Park SH, Park YS, Lim HY, Kang WK, Park JO. Triptolide as a novel agent in pancreatic cancer: the validation using patient derived pancreatic tumor cell line. BMC Cancer. 2018;18:1103.

35. Reno TA, Kim JY, Raz DJ. Triptolide inhibits lung cancer cell migration, invasion, and metastasis. Ann Thorac Surg. 2015;100:18171824; discussion 1824-1825.

36. Banerjee S, Saluja A. Minnelide, a novel drug for pancreatic and liver cancer. Pancreatology. 2015;15(4 Suppl):S39-S43.

37. Chugh R, Sangwan V, Patil SP, Dudeja V, Dawra RK, Banerjee S, Schumacher RJ, Blazar BR, Georg GI, Vickers SM, Saluja AK. A preclinical evaluation of Minnelide as a therapeutic agent against pancreatic cancer. Sci Transl Med. 2012;4:156ra139.

38. Giri B, Gupta VK, Yaffe B, Modi S, Roy P, Sethi V, Lavania SP, Vickers SM, Dudeja V, Banerjee S, Watts J, Saluja A. Pre-clinical evaluation of Minnelide as a therapy for acute myeloid leukemia. J Transl Med. 2019;17:163. 
39. Ramakrishnan V, de Haydu C, Wilkinson P, Hooda U, Giri B, Oleas JM, Rive V, Roy S, Dudeja V, Slomovitch B, Saluja A, Ramakrishnan $S$. Minnelide, a prodrug, inhibits cervical cancer growth by blocking HPV-induced changes in p53 and pRb. Am J Cancer Res. 2021;11:2202-2214.

40. Rivard C, Geller M, Schnettler E, Saluja M, Vogel RI, Saluja A, Ramakrishnan S. Inhibition of epithelial ovarian cancer by Minnelide, a water-soluble pro-drug. Gynecol Oncol. 2014;135:318-324.

41. Ding YY, Li JM, Guo FJ, Liu Y, Tong YF, Pan XC, Lu XL, Ye W, Chen XH, Zhang HG. Triptolide upregulates myocardial forkhead helix transcription factor p3 expression and attenuates cardiac hypertrophy. Front Pharmacol. 2016;7:471.

42. Li W, Gong K, Ding Y, Chaurasiya B, Ni Y, Wu Y, Zhao P, Shen Y, Zhang Z, Webster TJ. Effects of triptolide and methotrexate nanosuspensions on left ventricular remodeling in autoimmune myocarditis rats. Int J Nanomedicine. 2019;14:851-863.

43. Liu M, Chen J, Huang Y, Ke J, Li L, Huang D, Wu W. Triptolide alleviates isoprenaline-induced cardiac remodeling in rats via TGF- $\beta 1 /$ Smad3 and p38 MAPK signaling pathway. Pharmazie. 2015;70:244250.

44. Ge T, Qin H, Wang X, Yang SS, Guo L, Han W, Chang HY. Effects of thoracic epidural anesthesia on cardiac function and myocardial cell apoptosis in isoproterenol-induced chronic heart failure rats. J Interv Cardiol. 2014;27:446-455.

45. Liang Z, Leo S, Wen H, Ouyang M, Jiang W, Yang K. Triptolide improves systolic function and myocardial energy metabolism of diabetic cardiomyopathy in streptozotocin-induced diabetic rats. BMC Cardiovasc Disord. 2015;15:42.

46. Liu Y, Liu PP, Liu L, Zheng XS, Zheng H, Yang CC, Luobu CR, Liu Y. Triptolide inhibits TGF- $\beta$-induced matrix contraction and fibronectin production mediated by human Tenon fibroblasts. Int $J$ Ophthalmol. 2018;11:1108-1113.

47. Li XY, Wang SS, Han Z, Han F, Chang YP, Yang Y, Xue M, Sun B,
Chen LM. Triptolide restores autophagy to alleviate diabetic renal fibrosis through the miR-141-3p/PTEN/Akt/mTOR pathway. Mol Ther Nucleic Acids. 2017;9:48-56.

48. Fang YY, Wan L, Dong WZ, Wen JT, Liu J. [Effect of triptolide in improving platelet activation in patients with ankylosing spondylitis by regulating VEGFA,SDF-1,CXCR4 pathway]. Zhongguo Zhong Yao Za Zhi. 2019;44:3520-3525. Chinese.

49. Song C, Wang Y, Cui L, Yan F, Shen S. Triptolide attenuates lipopolysaccharide-induced inflammatory responses in human endothelial cells: involvement of NF- $\mathrm{B}$ pathway. BMC Complement Altern Med. 2019;19:198.

50. Martínez GJ, Celermajer DS, Patel S. The NLRP3 inflammasome and the emerging role of colchicine to inhibit atherosclerosis-associated inflammation. Atherosclerosis. 2018;269:262-271.

51. Li R, Lu K, Wang Y, Chen M, Zhang F, Shen H, Yao D, Gong K, Zhang Z. Triptolide attenuates pressure overload-induced myocardial remodeling in mice via the inhibition of NLRP3 inflammasome expression. Biochem Biophys Res Commun. 2017;485:69-75.

52. He L, Peng X, Liu G, Tang C, Liu H, Liu F, Zhou H, Peng Y. Antiinflammatory effects of triptolide on IgA nephropathy in rats. Immunopharmacol Immunotoxicol. 2015;37:421-427.

53. Wang L, Zhang L, Hou Q, Zhu X, Chen Z, Liu Z. Triptolide attenuates proteinuria and podocyte apoptosis via inhibition of NF- $\kappa \mathrm{B} /$ GADD45B. Sci Rep. 2018;8:10843.

54. Xie S, Deng W, Chen J, Wu QQ, Li H, Wang J, Wei L, Liu C, Duan M, Cai Z, Xie Q, Hu T, Zeng X, Tang Q. Andrographolide protects against adverse cardiac remodeling after myocardial infarction through enhancing Nrf2 signaling pathway. Int J Biol Sci. 2020;16:12-26.

55. Xi C, Peng S, Wu Z, Zhou Q, Zhou J. Toxicity of triptolide and the molecular mechanisms involved. Biomed Pharmacother. 2017;90:531-541. 